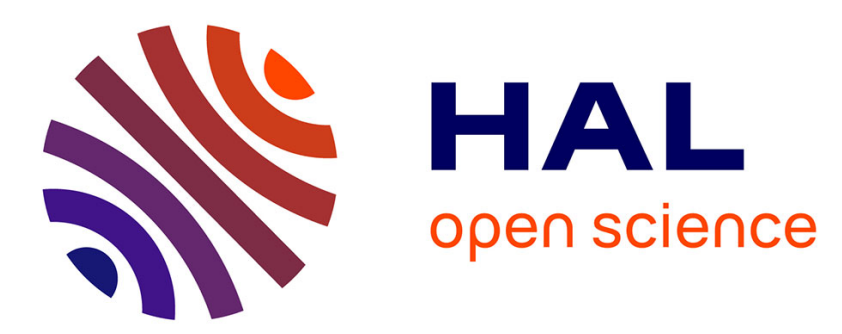

\title{
A piecewise affine control Lyapunov function for robust control
}

\author{
Ngoc Anh Nguyen, Sorin Olaru
}

\section{To cite this version:}

Ngoc Anh Nguyen, Sorin Olaru. A piecewise affine control Lyapunov function for robust control. 16th European Control Conference (ECC 2018), Jun 2018, Limassol, Cyprus. 10.23919/ecc.2018.8550436 . hal-01720263

\section{HAL Id: hal-01720263 \\ https://hal-centralesupelec.archives-ouvertes.fr/hal-01720263}

Submitted on 1 Mar 2018

HAL is a multi-disciplinary open access archive for the deposit and dissemination of scientific research documents, whether they are published or not. The documents may come from teaching and research institutions in France or abroad, or from public or private research centers.
L'archive ouverte pluridisciplinaire HAL, est destinée au dépôt et à la diffusion de documents scientifiques de niveau recherche, publiés ou non, émanant des établissements d'enseignement et de recherche français ou étrangers, des laboratoires publics ou privés. 


\title{
A piecewise affine control Lyapunov function for robust control
}

\author{
Ngoc Anh Nguyen ${ }^{1}$, Sorin Olaru ${ }^{1}$
}

\begin{abstract}
This paper presents the construction of a convex piecewise affine control Lyapunov function for constrained linear discrete-time systems, affected by bounded additive disturbances. Exploiting the properties of this control Lyapunov function, the closed-loop dynamics are shown to converge to a given full-dimensional robust positively invariant set. Moreover, the proposed method leads to a simple robust control algorithm which only requires solving a linear programming problem at each sampling instant. Finally, the controller design is illustrated via a numerical example.
\end{abstract}

\section{INTRODUCTION}

Lyapunov stability stands for a fundamental concept in control theory [23], since it has been applied in intensive studies related to both stability analysis and control design. Accordingly, control Lyapunov functions are usually employed to design controllers ensuring closed-loop stability in the sense of Lyapunov [20], [39]. Such control Lyapunov functions are usually chosen a priori with special properties. In the case of linear optimal control, suitable quadratic cost functions represent common control Lyapunov candidates, see for instance [1], [8], [10]. Also, polynomial control Lyapunov functions are used for linear/bilinear systems with nonlinear control laws in [37], [38]. Moreover, model predictive control (MPC) usually employs infinite horizon quadratic control Lyapunov functions, as shown in [9], [22], [24]. In case the underlying system is subject to constraints, such control Lyapunov functions should be determined such that the recursive feasibility is ensured. This problem is closely related to determining the feasible region.

One of the first studies on piecewise linear control Lyapunov functions for linear systems was presented in [16] for the nominal case, this result was subsequently extended for the robust case to cope with bounded additive disturbances and/or polytopic uncertainties in [6], [26], [35]. Although these studies lead to simple design formulations as linear programming problems, using these piecewise linear control Lyapunov functions cannot however provide explicitly the convergence region over which these functions are no longer strictly decreasing along the trajectories. Such a convergence region is usually known to be a robust positively invariant set. On the other hand, the recent study in [32] shows that a suitable convex lifting can ensure the convergence of the closed-loop dynamics to a given full-dimensional robust positively invariant set. Unfortunately, the closed-loop stability shown in this reference is not characterized in the sense

Ngoc Anh Nguyen, Sorin Olaru are with the Laboratory of Signals and Systems (L2S, UMR CNRS 8506), CentraleSupélec-CNRS-UPS, Université Paris Saclay, 3 rue Joliot Curie, Plateau de Moulon, 91192, Gif-sur-Yvette, France. Ngocanh.Nguyen.rs@gmail.com, Sorin.olaru@centralesupelec.fr of Lyapunov. In this paper, these drawbacks are resolved by means of a suitable construction of control Lyapunov function. More precisely, we present the construction of a convex piecewise affine control Lyapunov function which is more general than the piecewise linear family. Also, the constructed control Lyapunov function is able to ensure that the closed-loop dynamics converge to a given fulldimensional robust positively invariant set as time tends to infinity, leading to a simple control design procedure which only requires solving a linear program at each sampling instant. This result could be useful for systems with fast dynamics, e.g. active vibration system [14], [15].

\section{Generalities and basic notions}

Throughout the paper, $\mathbb{R}, \mathbb{N}, \mathbb{N}_{>0}$ denote the field of real numbers, the set of nonnegative integers and the positive integer set, respectively. The following index set is also defined for a given $N \in \mathbb{N}_{>0}: \mathcal{I}_{N}=\{1,2, \ldots, N\}$. A polyhedron is defined as the intersection of finitely many closed halfspaces. A polytope is defined as a bounded polyhedron. Also, $\mathcal{V}(P)$ denotes the set of vertices of polytope $P$. We use $\operatorname{int}(S)$ to denote the interior of a full-dimensional set $S$, while $\operatorname{conv}(S)$ denotes the convex hull of $S$. Given two sets $S_{1}, S_{2} \subset \mathbb{R}^{d}$, by $S_{1} \backslash S_{2}$, we denote the following set: $S_{1} \backslash S_{2}=\left\{x \in \mathbb{R}^{d}: x \in S_{1}, x \notin S_{2}\right\}$. Also, the Minkowski sum of these two sets, denoted by $S_{1} \oplus S_{2}$, is defined as follows: $S_{1} \oplus S_{2}=\left\{x_{1}+x_{2}: x_{1} \in S_{1}, x_{2} \in S_{2}\right\}$.

\section{Problem Settings}

In this paper, we consider a linear time-invariant system, affected by additive disturbances:

$$
x_{k+1}=A x_{k}+B u_{k}+w_{k},
$$

where $x_{k}, u_{k}, w_{k}$ denote the state, control variable and additive disturbance at time $k$. These variables satisfy

$$
x_{k} \in \mathbb{X}, u_{k} \in \mathbb{U}, w_{k} \in \mathbb{W},
$$

where the constraint sets $\mathbb{X} \subset \mathbb{R}^{d_{x}}, \mathbb{U} \subset \mathbb{R}^{d_{u}}, \mathbb{W} \subset \mathbb{R}^{d_{x}}$ are assumed to be polytopes, containing the origin in their interior, for given $d_{x}, d_{u} \in \mathbb{N}_{>0}$. This system is assumed to satisfy the following assumption.

Assumption 1: The pair $(A, B)$ is controllable.

In the sequel, the aim is to construct a control Lyapunov function, usually referred to as a convex lifting [27]. More clearly, the control Lyapunov function presented in this paper represents the convex and piecewise affine properties, which is more general than the piecewise linear family as in [6], [26], [35]. Furthermore, this control Lyapunov function leads to a result stronger than the one presented in the above 
references, i.e., the closed-loop dynamics converge to a given full-dimensional robust positively invariant set as time tends to infinity.

\section{CONSTRUCTION OF CONTROL LyAPUNOV FUNCTION}

Before presenting the main result, several important concepts will be recalled. They are instrumental in the proposed construction of control Lyapunov function. Positive invariance concept has been investigated in many studies [3]-[5], [7] and has been used in different control strategies. In case the system is affected by disturbances, the robust positive invariance concept is of use instead.

Definition 3.1: Given an admissible control law $u=$ $K x \in \mathbb{U}$, a set $\Omega \subseteq \mathbb{X}$ is called robust positively invariant with respect to system (1) subject to constraint (2) iff

$$
(A+B K) \Omega \oplus \mathbb{W} \subseteq \Omega .
$$

Such a controller $u=K x \in \mathbb{U}$ can be obtained by solving the discrete-time algebraic Riccati equation. This controller is only used to obtain a robust positively invariant set via existing algorithms [12], [21], [26], [36], which is an important element for the construction of a control Lyapunov function in this paper.

Another important component for the proposed construction is the feasible region. In this paper, we restrict our attention to a $\lambda$-contractive set for a given $\lambda \in[0,1)$. Its definition is recalled below.

Definition 3.2: Consider system (1) subject constraint (2). A set $\mathcal{X} \subseteq \mathbb{X}$ is called $\lambda$-contractive for a given $0 \leq \lambda<$ 1 , if there exists a control law $u=\kappa(x) \in \mathbb{U}$ such that $(A x+B \kappa(x)) \oplus \mathbb{W} \subseteq \lambda \mathcal{X}, \forall x \in \mathcal{X}$.

The maximal $\lambda$-contractive set, denoted as $P_{\lambda}$, is defined as the smallest set in $\mathbb{X}$ containing all the $\lambda$-contractive sets in $\mathbb{X}$. Algorithms for the computation of the maximal $\lambda$-contractive set are referred to [6], [19]. Throughout the rest of this paper, we will use $P_{\lambda}$ as the feasible region and $\Omega$ as the given robust positively invariant set. $P_{\lambda}$ and $\Omega$ are assumed to satisfy the following properties.

Assumption 2: $\Omega, P_{\lambda}$ are full-dimensional polytopes and satisfy $\Omega \subset P_{\lambda}$.

It is worth emphasizing that Assumption 2 is not restrictive, since one can choose a value of $\lambda \in[0,1)$ sufficiently large to satisfy $\Omega \subset P_{\lambda}$. Given a robust positively invariant set $\Omega$ and a constant $\delta>0$, we define the following elements:

$$
\begin{aligned}
\widehat{V}_{0} & :=\left\{\left[\begin{array}{ll}
v^{T} & \delta
\end{array}\right]^{T}: v \in \mathcal{V}(\Omega)\right\} \cup\{0\} \subset \mathbb{R}^{d_{x}+1}, \\
\widehat{\Pi}_{0} & :=\operatorname{conv}\left(\widehat{V}_{0}\right), \\
\tau(x) & :=\arg \min _{z} z \text { s.t. }\left[x^{T} z\right]^{T} \in \widehat{\Pi}_{0} .
\end{aligned}
$$

Important properties of function $\tau(x)$, defined in (3), are presented in the following lemma.

Lemma 3.1: $\tau(x)$, defined in (3),

1) is a convex, continuous, piecewise affine function;

2) satisfies $0 \leq \tau(x) \leq \delta$ for all $x \in \Omega$;

3) satisfies $\tau(x)=0$ only if $x=0$;

4) satisfies $\tau(x)=\delta$ for $x \in \mathcal{V}(\Omega)$.

The proof is referred to Subsection VII-A for reading ease.
Remark 3.1: We remark that one can choose any value of constant $\delta>0$ to construct $\tau(x)$ as in (3) without affecting its properties shown in Lemma 3.1.

We now construct a control Lyapunov function $\ell(x)$ defined over the maximal $\lambda$-contractive set. As will be clear later, function $\ell(x)$ is required to be convex, piecewise affine and its values over the vertices of $P_{\lambda}$ not belonging to $\Omega$ are identical. More precisely, we need to give a suitable height $h$ to the vertices of $P_{\lambda}$ such that $\ell(v)=h$ for all $v \in \mathcal{V}\left(P_{\lambda}\right) \backslash \Omega$. Furthermore, function $\ell(x)$ should satisfy $\ell(x)=\tau(x)$ for all $x \in \Omega$. These requirements will be of use later to prove the strict decrease of $\ell(x)$ along the trajectories in $P_{\lambda} \backslash \Omega$.

As proven in Lemma 3.1, $\tau(x)$ defined in (3), is a piecewise affine function, thus let $\left\{\Omega_{j}\right\}_{j \in \mathcal{I}_{M}}$ denote the polytopic partition of $\Omega$ associated with $\tau(x)$ (the definition of a polyhedral/polytopic partition is referred to [31]) and we denote $\tau(x)$ as follows:

$$
\tau(x)=a_{j}^{T} x+b_{j} \text { for } x \in \Omega_{j},
$$

where $a_{j} \in \mathbb{R}^{d_{x}}, b_{j} \in \mathbb{R}, j \in \mathcal{I}_{M}$ for a suitable $M \in \mathbb{N}_{>0}$. Define also the following function:

$$
\widehat{\tau}(x):=\max _{j \in \mathcal{I}_{M}} a_{j}^{T} x+b_{j} \text { for all } x \in \mathbb{R}^{d_{x}} .
$$

Obviously, function $\widehat{\tau}(x)$ is convex over $\mathbb{R}^{d_{x}}$ by its definition. Moreover, this function possesses the following property.

Lemma 3.2: Function $\widehat{\tau}(x)$, defined in (5), satisfies $\widehat{\tau}(x)=\tau(x)$ for all $x \in \Omega$.

Proof: As proven in Lemma 3.1, $\tau(x)$ is a convex, piecewise affine function over $\Omega$, accordingly it can also be represented by:

$$
\tau(x)=\max _{j \in \mathcal{I}_{M}} a_{j}^{T} x+b_{j} \text { for all } x \in \Omega .
$$

Therefore, the definition of $\widehat{\tau}(x)$ in (5) satisfies $\widehat{\tau}(x)=\tau(x)$ for any $x \in \Omega$.

The construction of a control Lyapunov candidate $\ell(x)$ over $P_{\lambda}$, satisfying the above requirements, is put forward in the sequel with respect to a given constant $\epsilon>0$ :

$$
\begin{aligned}
h & :=\min _{z} z \text { s.t. } \widehat{\tau}(v)+\epsilon \leq z, \forall v \in \mathcal{V}\left(P_{\lambda}\right) \backslash \Omega, \\
\widehat{V} & :=\left\{\left[v^{T} h\right]^{T}: v \in \mathcal{V}\left(P_{\lambda}\right)\right\} \cup \widehat{V}_{0}, \\
\widehat{\Pi} & :=\operatorname{conv}(\widehat{V}), \\
\ell(x) & :=\arg \min _{z} z \text { s.t. }\left[x^{T} z\right]^{T} \in \widehat{\Pi} .
\end{aligned}
$$

The construction of $\ell(x)$ in (7) requires solving parametric linear programming problems, which can be performed by means of existing algorithms in [2], [13], [33], [34]. This function represents the following properties.

Lemma 3.3: Function $\ell(x)$ defined in (7) is convex, continuous and piecewise affine.

Proof: The proof follows similar arguments of claim 1) in Lemma 3.1.

For ease of presentation, let $\left\{\mathcal{X}_{i}\right\}_{i \in \mathcal{I}_{N}}$ for a suitable $N \in$ $\mathbb{N}_{>0}$ denote the polytopic partition of $P_{\lambda}$ associated with function $\ell(x)$. It can also be proven that function $\ell(x)$ represents a convex lifting for the polytopic partition $\left\{\mathcal{X}_{i}\right\}_{i \in \mathcal{I}_{N}}$. 
The interested reader is referred to [27]-[29] for further details about the definition of convex lifting and the proof of the above observation.

We now prove that function $\ell(x)$, defined in (7), satisfies the aforementioned requirements. This is formally stated in the following result.

Lemma 3.4: Function $\ell(x), h, \delta$ satisfy:

1) $h>\delta>0$

2) $0 \leq \ell(x) \leq h$ for all $x \in P_{\lambda}$;

3) $\ell(x)=h$ for all $x \in \mathcal{V}\left(P_{\lambda}\right) \backslash \Omega$;

4) $\ell(x)=\tau(x)$ for all $x \in \Omega$;

5) $\ell(x)>0$ for all $x \in P_{\lambda} \backslash\{0\}$;

6) $\ell(x)>\delta$ for $x \in P_{\lambda} \backslash \Omega$;

7) $\ell(\beta x) \leq \beta \ell(x)$ for all $0 \leq \beta \leq 1$ and all $x \in P_{\lambda}$.

The proof is presented in Subsection VII-B.

Remark 3.2: Note that any value of $h$ larger than the value obtained in (7) does not affect the properties of function $\ell(x)$ shown in Lemma 3.4.

\section{Control DESign PROCEDURE}

The construction and prominent properties of a control Lyapunov candidate have been presented in the preceding section. In this section, we prove that function $\ell(x)$ defined in (7) satisfies the properties of a control Lyapunov function. Definition of a local input-to-state stability (ISS) Lyapunov function is referred to [18], [20]. Note that since $\ell(x)$ satisfies the properties shown in Lemma 3.4, there always exist two suitable constants $c_{2}>c_{1}>0$ such that

$$
c_{1}\|x\|_{\infty} \leq \ell(x) \leq c_{2}\|x\|_{\infty} \text { for all } x \in P_{\lambda} ;
$$

the proof is referred to Lemma 5.1 in [30]. Therefore, to prove $\ell(x)$ to be a control Lyapunov function, one needs to show that there exists a controller $u=\kappa(x) \in \mathbb{U}$ for all $x \in P_{\lambda}$ such that $\ell(x)$ is strictly decreasing along the trajectories outside the given robust positively invariant set $\Omega$. Such a controller is put forward in Algorithm 1.

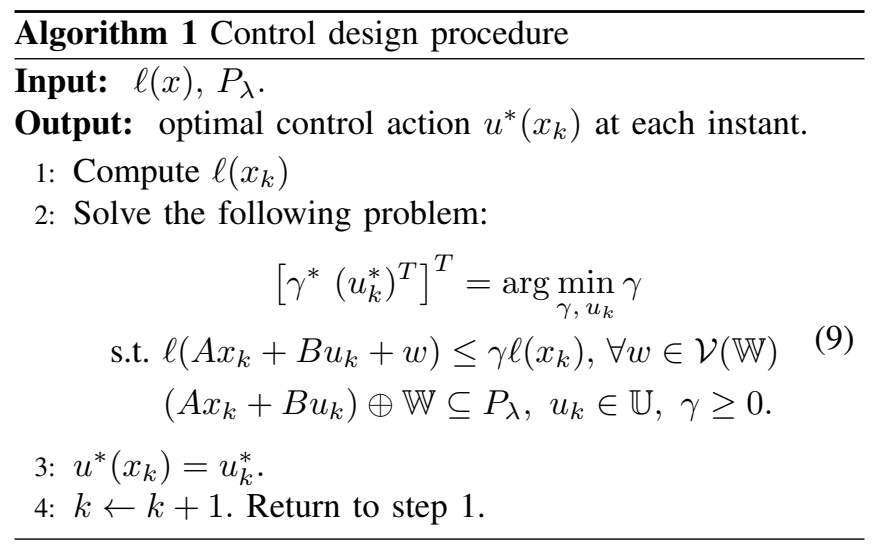

We now return to prove that the convex lifting $\ell(x)$ defined in (7) and the controller designed in Algorithm 1 satisfy the strict decrease of $\ell(x)$ along the trajectories outside $\Omega$. This is formally stated in the following proposition.
Proposition 4.1: Consider function $\ell(x)$ defined in (7) and the controller designed in Algorithm 1, then for any $x \in$ $P_{\lambda} \backslash \Omega$, it satisfies:

$$
\ell\left(A x+B u^{*}(x)+w\right)<\ell(x), \forall w \in \mathbb{W} .
$$

Proof: Consider a vertex $v \in \mathcal{V}\left(P_{\lambda}\right) \backslash \Omega$. According to the definition of $P_{\lambda}$ in Definition 3.2, there exists a control action, denoted by $u(v) \in \mathbb{U}$, such that

$$
A v+B u(v)+w \in \lambda P_{\lambda}, \forall w \in \mathbb{W} .
$$

Accordingly, for each $w \in \mathbb{W}$, there exists a point $y(w) \in P_{\lambda}$ such that $A v+B u(v)+w=\lambda y(w)$. Claims 7) and 3) of Lemma 3.4 yield

$$
\ell(A v+B u(v)+w) \leq \lambda \ell(y(w)) \leq \lambda h=\lambda \ell(v) .
$$

Otherwise, if $v \in \mathcal{V}\left(P_{\lambda}\right) \cap \Omega$, there exists a control action, also denoted by $u(v) \in \mathbb{U}$, such that $A v+B u(v)+w \in \Omega$ for all $w \in \mathbb{W}$, since $\Omega$ is robust positively invariant. According to claim 4) of Lemma 3.4 and Lemma 3.1, we obtain

$$
\ell(A v+B u(v)+w) \leq \delta=\ell(v) .
$$

It can be observed that for any $x \in P_{\lambda} \backslash \Omega$, there exists a region $\mathcal{X}_{i}$ of the polytopic partition $\left\{\mathcal{X}_{i}\right\}_{i \in \mathcal{I}_{N}}$ of $P_{\lambda}$, associated with $\ell(x)$ such that $x \in \mathcal{X}_{i}$. As a consequence, $x$ can be written in the form:

$$
x=\sum_{v \in \mathcal{V}\left(\mathcal{X}_{i}\right)} \alpha(v) v, \alpha(v) \geq 0, \sum_{v \in \mathcal{V}\left(\mathcal{X}_{i}\right)} \alpha(v)=1 .
$$

As $x \in P_{\lambda} \backslash \Omega$, there exists at least one vertex $v \in \mathcal{V}\left(\mathcal{X}_{i}\right) \backslash \Omega$ such that $\alpha(v)>0$ and subsequently $\alpha(v) \ell(v)>0$, leading to the following:

$$
\begin{aligned}
\ell(x) & =\sum_{v \in \mathcal{V}\left(\mathcal{X}_{i}\right)} \alpha(v) \ell(v) \\
& >\sum_{v \in \mathcal{V}\left(\mathcal{X}_{i}\right) \backslash \Omega} \alpha(v) \lambda \ell(v)+\sum_{v \in \mathcal{V}\left(\mathcal{X}_{i}\right) \cap \Omega} \alpha(v) \ell(v) .
\end{aligned}
$$

Note that inclusion (14a) is due to the fact that $\ell(x)$ is affine over $\mathcal{X}_{i}$. On the other hand, inclusions (11) and (12) lead to

$$
\begin{aligned}
\sum_{v \in \mathcal{V}\left(\mathcal{X}_{i}\right) \backslash \Omega} \alpha(v) \lambda \ell(v)+\sum_{v \in \mathcal{V}\left(\mathcal{X}_{i}\right) \cap \Omega} \alpha(v) \ell(v) \\
\geq \sum_{v \in \mathcal{V}\left(\mathcal{X}_{i}\right)} \alpha(v) \ell(A v+B u(v)+w) .
\end{aligned}
$$

According to the convexity of $\ell(x)$, shown in Lemma 3.3, one obtains:

$$
\begin{aligned}
& \sum_{v \in \mathcal{V}\left(\mathcal{X}_{i}\right)} \alpha(v) \ell(A v+B u(v)+w) \\
& \geq \ell\left(A x+B \sum_{v \in \mathcal{V}\left(\mathcal{X}_{i}\right)} \alpha(v) u(v)+w\right) .
\end{aligned}
$$

It is observed that $u(v) \in \mathbb{U}$ for all $v \in \mathcal{V}\left(\mathcal{X}_{i}\right)$, therefore the convexity of $\mathbb{U}$ yields $\sum_{v \in \mathcal{V}\left(\mathcal{X}_{i}\right)} \alpha(v) u(v) \in \mathbb{U}$, and subsequently the following inclusion:

$$
\begin{aligned}
\ell\left(A x+B \sum_{v \in \mathcal{V}\left(\mathcal{X}_{i}\right)} \alpha(v) u(v)+w\right) & \\
& \geq \ell\left(A x+B u^{*}(x)+w\right) .
\end{aligned}
$$


Finally, incorporating the inclusions (14a)-(17), we conclude that $\ell(x)>\ell\left(A x+B u^{*}(x)+w\right), \forall w \in \mathbb{W}, \forall x \in P_{\lambda} \backslash \Omega$. The proof is complete.

We now investigate the behavior of the closed-loop dynamics if $x \in \Omega$.

Proposition 4.2: Consider function $\ell(x)$ defined in (7) and the controller designed in Algorithm 1, then any point $x \in \Omega$ satisfies

$$
\ell\left(A x+B u^{*}(x)+w\right) \leq \ell(x)+\ell(w), \forall w \in \mathbb{W} .
$$

Proof: As $x \in \Omega$, there exists a region $\Omega_{j}$ in the polytopic partition $\left\{\Omega_{j}\right\}_{j \in \mathcal{I}_{M}}$ of $\Omega$, associated with $\tau(x)$, such that $x \in \Omega_{j}$. Region $\Omega_{j}$ has its vertices as vertices of $\Omega$ and the origin. Therefore, $x$ can be written as follows:

$$
x=\sum_{v \in \mathcal{V}\left(\Omega_{j}\right)} \alpha(v) v, \alpha(v) \geq 0, \sum_{v \in \mathcal{V}\left(\Omega_{j}\right)} \alpha(v)=1 .
$$

Also, since $\Omega$ represents a robust positively invariant set, then for any $v \in \mathcal{V}(\Omega)$, there exists a control action $u(v) \in \mathbb{U}$ such that $A v+B u(v)+w \in \Omega$ for all $w \in \mathbb{W}$. In other words, inclusion (12) holds for any $v \in \mathcal{V}(\Omega)$. Accordingly, claim 4) of Lemma 3.4 leads to:

$$
\begin{aligned}
\ell(x) & =\sum_{v \in \mathcal{V}\left(\Omega_{j}\right)} \alpha(v) \ell(v) \\
& \geq \sum_{v \in \mathcal{V}\left(\Omega_{j}\right) \backslash\{0\}} \alpha(v) \ell(A v+B u(v)+w) .
\end{aligned}
$$

If one chooses $u(0)=0$, then inclusion (20) yields

$$
\begin{aligned}
\ell(x)+\ell(w) & \geq \ell(x)+\alpha(0) \ell(w) \\
& \geq \sum_{v \in \mathcal{V}\left(\Omega_{j}\right)} \alpha(v) \ell(A v+B u(v)+w) \\
& \geq \ell\left(A x+B \sum_{v \in \mathcal{V}\left(\Omega_{j}\right)} \alpha(v) u(v)+w\right) \\
& \geq \ell\left(A x+B u^{*}(x)+w\right) .
\end{aligned}
$$

Note that inclusion (21b) follows the convexity of $\ell(x)$ over $P_{\lambda}$, while inclusion (21c) is derived from the convexity of $\mathbb{U}$ and $\sum_{v \in \mathcal{V}\left(\Omega_{j}\right)} \alpha(v) u(v) \in \mathbb{U}$.

The main result of the paper is formally stated in the following theorem.

Theorem 4.3: Given system (1) subject to constraint (2), the controller designed in Algorithm 1 guarantees the recursive feasibility and input-to-state stability.

Proof: The recursive feasibility is guaranteed by suitable $\gamma$ according to Propositions 4.1 and 4.2. Also, Proposition 4.1 shows that $\left\{\ell\left(x_{k}\right)\right\}_{k=0}^{\infty}$ is strictly decreasing outside $\Omega$ and bounded in $[\delta, h]$, therefore $\lim _{k \rightarrow \infty} \ell\left(x_{k}\right)=\delta$. Roughly speaking, $x_{k}$ converges to $\Omega$ as time tends to infinity, leading to the input-to-state stability in the sense of Lyapunov.

Remark 4.1: It is observed that inside $\Omega$ the closed-loop dynamics still continue converging to the minimal robust positively invariant set with the same controller.

Remark 4.2: Since $\ell(x)$ represents a convex piecewise affine function, then formulation (9) is a linear program. Accordingly, this control Lyapunov function leads to a simple control algorithm by only requiring the resolution of a linear programming problem at each sampling instant. Note also that explicit solution of (9) can be obtained and be implemented by means of the convex lifting concept as presented in [27]. However, the proposed method is rather convenient for small-dimensional systems like most of existing settheoretic methods.

\section{NUMERICAL EXAMPLE}

To illustrate the proposed method, we consider the following model of a vibration attenuation system [15]:

$$
x_{k+1}=\left[\begin{array}{cc}
0.5269 & 0.0166 \\
-43.0741 & 0.5182
\end{array}\right] x_{k}+\left[\begin{array}{c}
-0.0011 \\
-0.098
\end{array}\right] u_{k}+w_{k} .
$$

Also, the state, control variable and disturbance are subject to the following constraints:

$$
-\left[\begin{array}{c}
10 \\
200
\end{array}\right] \leq x_{k} \leq\left[\begin{array}{c}
10 \\
200
\end{array}\right],\left\|u_{k}\right\|_{\infty} \leq 100,\left\|w_{k}\right\|_{\infty} \leq 0.1 .
$$

We choose the following controller $u=$ $\left[\begin{array}{ll}-21.3982 & 0.7397\end{array}\right] x$ to compute a robust positively invariant set $\Omega$. Accordingly, the maximal output admissible set $\Omega$ is computed and shown in Fig. 1, where the maximal 0.999-contractive set $P_{0.999}$ satisfying Assumption 2 is also presented. One can also choose the minimal robust positively invariant set for $\Omega$ to ensure a smaller impact of disturbances. On the other hand, a control Lyapunov function, computed with $\delta=1, \epsilon=10^{-5}$, is shown in Fig. 2. It is shown therein that the controller designed in Algorithm 1 makes $\ell(x)$ strictly decreasing along the trajectories outside $\Omega$, leading to closed-loop stability in the sense of Lyapunov. The convergence of closed-loop dynamics to $\Omega$ is more clearly illustrated in Fig. 1. It is worth emphasizing that Algorithm 1 is composed of 36 constraints in this example, while MPC method [25] with a quadratic cost function requires 62 constraints for the prediction horizon 10; an MPC problem with a $1 / \infty$-norm cost function requires even more constraints. Finally, the numerical example of this paper is simulated in the environment of MPT 3.0 [17].

\section{CONCLUSIONS}

This paper presented a method to construct a convex, piecewise affine control Lyapunov function for linear systems affected by bounded additive disturbances. This control Lyapunov function was shown to ensure that the closedloop dynamics converge to a given robust positively invariant set. Also, it was shown to lead to a simple control design procedure as only requiring the resolution of a linear program at each sampling instant. Finally, the control design was illustrated via a numerical example.

\section{APPENDIX}

\section{A. Proof of Lemma 3.1}

1) is a direct consequence of Theorems IV-3 and IV-4 in [11]. For claim 2), it is observed that any point $\left[x^{T} z\right]^{T} \in$ 


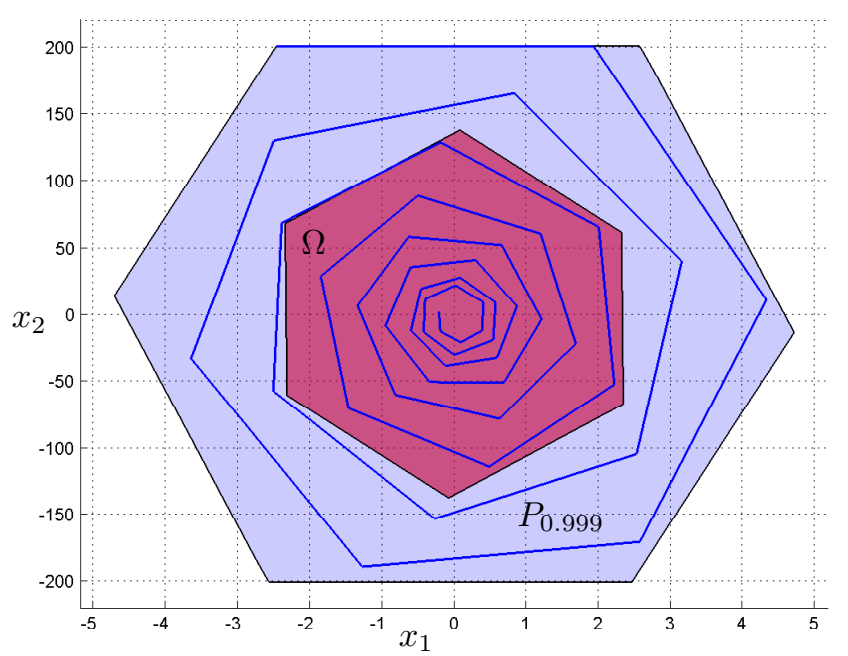

Fig. 1. Maximal output admissible set $\Omega$, the maximal 0.999-contractive set $P_{0.999}$ and the closed-loop dynamics.

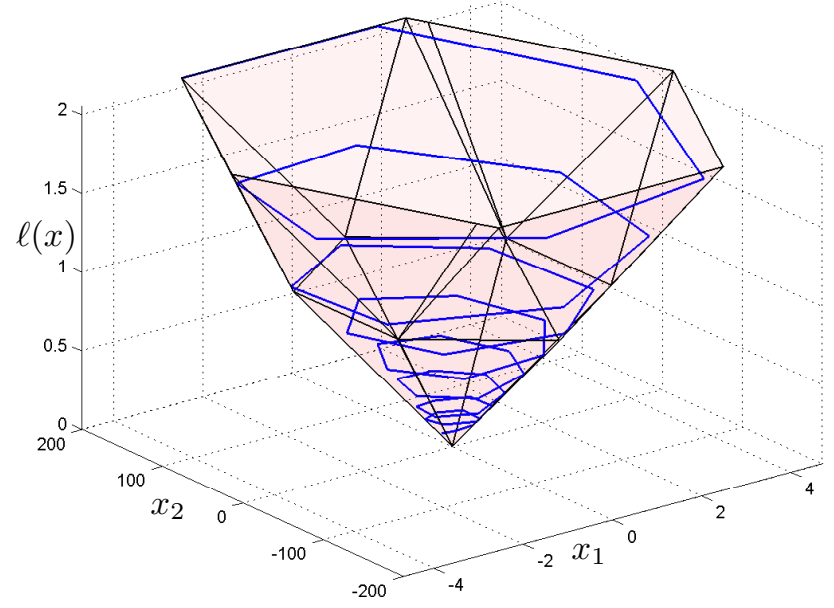

Fig. 2. The constructed control Lyapunov function with $\delta=1$ and $\epsilon=$ $10^{-5}$ and its strict decrease along the dynamics outside $\Omega$.

$\widehat{\Pi}_{0}$ can be expressed as a convex combination of the points in $\widehat{V}_{0}$, defined in (3), i.e.,

$$
\begin{gathered}
{\left[\begin{array}{ll}
x^{T} & z
\end{array}\right]^{T}=\alpha(0) 0+\sum_{v \in \mathcal{V}(\Omega)} \alpha(v)\left[\begin{array}{ll}
v^{T} & \delta
\end{array}\right]^{T}} \\
\alpha(0), \alpha(v) \geq 0, \alpha(0)+\sum_{v \in \mathcal{V}(\Omega)} \alpha(v)=1 .
\end{gathered}
$$

According to inclusion (22), claim 2) is easily deduced by the following argument:

$$
0 \leq z=\sum_{v \in \mathcal{V}(\Omega)} \alpha(v) \delta \leq \delta
$$

Also, the left equality in (23) holds only if $\alpha(v)=0$ for all $v \in \mathcal{V}(\Omega)$ and $\alpha(0)=1$, leading to claim 3). On the other hand, as $\tau(x)$ is convex over $\Omega$, then it attains its maximal value $\delta$ at the vertices of $\Omega$, leading to claim 4).

\section{B. Proof of Lemma 3.4}

Any point $x \in P_{\lambda}$ can be written as a convex combination of the vertices of $P_{\lambda}$, i.e., $x=\sum_{v \in \mathcal{V}\left(P_{\lambda}\right)} \alpha(v) v, \alpha(v) \geq 0$, $\sum_{v \in \mathcal{V}\left(P_{\lambda}\right)} \alpha(v)=1$. Accordingly, we obtain:

$$
\begin{gathered}
\sum_{v \in \mathcal{V}\left(P_{\lambda}\right) \backslash \Omega} \alpha(v) h+\sum_{v \in \mathcal{V}\left(P_{\lambda}\right) \cap \Omega} \alpha(v) \delta \\
\geq \sum_{v \in \mathcal{V}\left(P_{\lambda}\right) \backslash \Omega} \alpha(v) \epsilon+\sum_{v \in \mathcal{V}\left(P_{\lambda}\right)} \alpha(v) \widehat{\tau}(v) \\
\geq \sum_{v \in \mathcal{V}\left(P_{\lambda}\right) \backslash \Omega} \alpha(v) \epsilon+\widehat{\tau}(x) .
\end{gathered}
$$

Note that inclusion (24a) is obtained due to the definition in (7) and Lemma 3.2, while inclusion (24b) is due to the convexity of $\widehat{\tau}(x)$. As a consequence, if one chooses $x \in \mathcal{V}(\Omega)$ such that there exists at least one $v \in \mathcal{V}\left(P_{\lambda}\right) \backslash \Omega$ satisfying $\alpha(v)>0$, then we conclude that $h \geq \epsilon+\delta>\delta$, leading to claim 1).

For claim 2), let us consider any point $\left[x^{T} z\right]^{T} \in \widehat{\Pi}$, then it can be written in the following form:

$$
\begin{aligned}
& {\left[\begin{array}{l}
x \\
z
\end{array}\right]=\beta(0) 0+\sum_{v \in \mathcal{V}(\Omega)} \alpha_{1}(v)\left[\begin{array}{l}
v \\
\delta
\end{array}\right]+\sum_{v \in \mathcal{V}\left(P_{\lambda}\right)} \alpha_{2}(v)\left[\begin{array}{l}
v \\
h
\end{array}\right]} \\
& \beta(0)+\sum_{v \in \mathcal{V}(\Omega)} \alpha_{1}(v)+\sum_{v \in \mathcal{V}\left(P_{\lambda}\right)} \alpha_{2}(v)=1 \\
& \beta(0), \alpha_{1}(v), \alpha_{2}(v) \geq 0 .
\end{aligned}
$$

According to expression (25) and claim 1), we obtain:

$$
0 \leq z=\sum_{v \in \mathcal{V}(\Omega)} \alpha_{1}(v) \delta+\sum_{v \in \mathcal{V}\left(P_{\lambda}\right)} \alpha_{2}(v) h \leq h,
$$

leading to claim 2).

Since $\ell(x)$ is a convex function over $P_{\lambda}$, it attains its maximal value $h$, according to claim 2), at vertices of $P_{\lambda}$. However, any $x \in \mathcal{V}\left(P_{\lambda}\right) \cap \Omega$ satisfies $\ell(x) \leq \delta<h$, as $\alpha_{2}(v)=0$ for all $v \in \mathcal{V}\left(P_{\lambda}\right)$ also fulfill expression (25) for $x \in \Omega$. In other words, $\ell(x)=h$ for $x \in \mathcal{V}\left(P_{\lambda}\right) \backslash \Omega$, as stated in claim 3 ).

To prove claim 4), we note that

$$
\begin{aligned}
& h \geq \epsilon+\delta \geq \epsilon+\widehat{\tau}(v) \text { for } v \in \mathcal{V}\left(P_{\lambda}\right) \cap \Omega \\
& h \geq \epsilon+\widehat{\tau}(v) \text { for } v \in \mathcal{V}\left(P_{\lambda}\right) \backslash \Omega
\end{aligned}
$$

where (27a) follows the proof of claim 1) and Lemma 3.2, while (27b) follows by its definition in (7). Accordingly, making use of expression (25) yields:

$$
\begin{aligned}
z & =\sum_{v \in \mathcal{V}(\Omega)} \alpha_{1}(v) \delta+\sum_{v \in \mathcal{V}\left(P_{\lambda}\right)} \alpha_{2}(v) h \\
& \geq \sum_{v \in \mathcal{V}(\Omega)} \alpha_{1}(v) \widehat{\tau}(v)+\sum_{v \in \mathcal{V}\left(P_{\lambda}\right)} \alpha_{2}(v)(\epsilon+\widehat{\tau}(v)) \\
& \geq \sum_{v \in \mathcal{V}\left(P_{\lambda}\right)} \alpha_{2}(v) \epsilon+\widehat{\tau}(x) \geq \widehat{\tau}(x) .
\end{aligned}
$$

It is worth noting that inclusion (28b) follows as a consequence of (27a) and (27b), also inclusion (28c) is obtained by the convexity of $\widehat{\tau}(x)$. We underline that inclusion (28c) 
becomes equality only if $\alpha_{2}(v)=0$ for all $v \in \mathcal{V}\left(P_{\lambda}\right)$, leading to $x \in \Omega$. Roughly speaking, $\ell(x)=\widehat{\tau}(x)=\tau(x)$ only if $x \in \Omega$, as stated in claim 4).

Claim 5) follows directly from inclusion (26), the lefthand side inequality becomes equality only if $\alpha_{1}(v)=0$ for all $v \in \mathcal{V}(\Omega), \alpha_{2}(v)=0$ for all $v \in \mathcal{V}\left(P_{\lambda}\right)$ and $\beta(0)=1$. Otherwise, $\ell(x)>0$ for any $x \in P_{\lambda} \backslash\{0\}$.

To prove claim 6), we note that for any $x \in P_{\lambda} \backslash \Omega$, there exists a region $\mathcal{X}_{j}$ of the polytopic partition $\left\{\mathcal{X}_{i}\right\}_{i \in \mathcal{I}_{N}}$ such that $x \in \mathcal{X}_{j}$ and $x=\sum_{v \in \mathcal{V}\left(\mathcal{X}_{j}\right)} \alpha(v) v, \alpha(v) \geq$ $0, \sum_{v \in \mathcal{V}\left(\mathcal{X}_{j}\right)} \alpha(v)=1$. As $x \in P_{\lambda} \backslash \Omega$ and $0 \in \operatorname{int}(\Omega)$, the vertices of $\mathcal{X}_{j}$ do not include the origin, leading to $\ell(v) \geq \delta$ for all $v \in \mathcal{V}\left(\mathcal{X}_{j}\right)$ because $\mathcal{V}\left(\mathcal{X}_{j}\right) \subseteq \mathcal{V}\left(P_{\lambda}\right) \cup \mathcal{V}(\Omega)$. Also, $x \notin \Omega$, there exists at least a vertex $v \in \mathcal{V}\left(\mathcal{X}_{j}\right) \backslash \Omega$ such that $\alpha(v)>0$ and consequently $\ell(v)=h>\delta$. As a consequence, it yields: $\ell(x)=\sum_{v \in \mathcal{V}\left(\mathcal{X}_{j}\right)} \alpha(v) \ell(v)>\sum_{v \in \mathcal{V}\left(\mathcal{X}_{j}\right)} \alpha(v) \delta=$ $\delta$, leading to claim 6 ).

Finally, claim 7) easily follows by the convexity of $\ell(x)$, as proven in Lemma 3.3, i.e., $\ell(\beta x) \leq \beta \ell(x)+(1-\beta) \ell(0)=$ $\beta \ell(x)$. The proof is complete.

\section{REFERENCES}

[1] B. D. Anderson and J. B. Moore, Optimal control: linear quadratic methods. Courier Corporation, 2007.

[2] M. Bemporad, A.and Morari, V. Dua, and E. N. Pistikopoulos, "The explicit linear quadratic regulator for constrained systems," Automatica, vol. 38, no. 1, pp. 3-20, 2002.

[3] G. Bitsoris, "On the positive invariance of polyhedral sets for discretetime systems," Systems \& Control Letters, vol. 11, no. 3, pp. 243-248, 1988.

[4] — , "Positively invariant polyhedral sets of discrete-time linear systems," International Journal of Control, vol. 47, no. 6, pp. 17131726, 1988.

[5] G. Bitsoris and M. Vassilaki, "Constrained regulation of linear systems," Automatica, vol. 31, no. 2, pp. 223-227, 1995.

[6] F. Blanchini, "Ultimate boundedness control for uncertain discretetime systems via set-induced Lyapunov functions," Automatic Control, IEEE Transactions on, vol. 39, no. 2, pp. 428-433, Feb 1994.

[7] F. Blanchini and S. Miani, Set-theoretic methods in control. Springer, 2007.

[8] D. Chmielewski and V. Manousiouthakis, "On constrained infinitetime linear quadratic optimal control," Systems \& Control Letters, vol. 29 , no. 3, pp. $121-129,1996$.

[9] F. A. Cuzzola, J. C. Geromel, and M. Morari, "An improved approach for constrained robust model predictive control," Automatica, vol. 38, no. 7, pp. 1183-1189, 2002.

[10] J. Daafouz and J. Bernussou, "Parameter dependent Lyapunov functions for discrete time systems with time varying parametric uncertainties," Systems \& Control Letters, vol. 43, no. 5, pp. 355-359, 2001.

[11] T. Gal, Postoptimal analyses, parametric programming and related topics. Walter de Gruyter, 1995.

[12] E. G. Gilbert and K. T. Tan, "Linear systems with state and control constraints: The theory and application of maximal output admissible sets," Automatic Control, IEEE Transactions on, vol. 36, no. 9, pp. 1008-1020, 1991

[13] A. Grancharova and T. A. Johansen, Explicit nonlinear model predictive control: theory and applications. Springer Science \& Business Media, 2012, vol. 429.

[14] M. Gulan, G. Takács, N. A. Nguyen, S. Olaru, P. Rodriguez-Ayerbe, and B. Rohal'-Ilkiv, "Efficient embedded model predictive vibration control via convex lifting," Control Systems Technology, IEEE Transactions on (to appear), 2017.

[15] _ _ "Embedded linear model predictive control for 8-bit microcontrollers via convex lifting," in the 20th IFAC World Congress, Toulouse, France, July 9-14 2017.

[16] P.-O. Gutman and M. Cwikel, "An algorithm to find maximal state constraint sets for discrete-time linear dynamical systems with bounded controls and states," Automatic Control, IEEE Transactions on, vol. 32, no. 3, pp. 251-254, 1987.
[17] M. Herceg, M. Kvasnica, C. Jones, and M. Morari, "Multi-parametric toolbox 3.0," in Control Conference (ECC), 2013 European, July 2013, pp. $502-510$.

[18] Z. P. Jiang and Y. Wang, "Input-to-state stability for discrete-time nonlinear systems," Automatica, vol. 37, no. 6, pp. 857-869, 2001.

[19] E. C. Kerrigan, "Robust constraint satisfaction: Invariant sets and predictive control," Ph.D. dissertation, University of Cambridge, 2001.

[20] H. K. Khalil, "Nonlinear systems," Prentice Hall, Inc, p. 323, 2002.

[21] I. Kolmanovsky and E. G. Gilbert, "Theory and computation of disturbance invariant sets for discrete-time linear systems," Mathematical Problems in Engineering, vol. 4, no. 4, pp. 317-367, 1998.

[22] M. V. Kothare, V. Balakrishnan, and M. Morari, "Robust constrained model predictive control using linear matrix inequalities," Automatica, vol. 32, no. 10, pp. 1361-1379, 1996.

[23] A. M. Lyapunov, "Problème général de la stabilité du mouvement," in Annales de la Faculté des Sciences de Toulouse, vol. 9. Université Paul Sabatier, 1907, pp. 203-474.

[24] D. Q. Mayne, J. B. Rawlings, C. V. Rao, and P. O. M. Scokaert, "Constrained model predictive control: Stability and optimality," $A u$ tomatica, vol. 36, no. 6, pp. 789-814, 2000.

[25] D. Q. Mayne, M. M. Seron, and S. V. Raković, "Robust model predictive control of constrained linear systems with bounded disturbances," Automatica, vol. 41, no. 2, pp. 219-224, 2005.

[26] H.-N. Nguyen, P.-O. Gutman, S. Olaru, and M. Hovd, "Implicit improved vertex control for uncertain, time-varying linear discretetime systems with state and control constraints," Automatica, vol. 49, no. 9, pp. 2754-2759, 2013.

[27] N. A. Nguyen, M. Gulan, S. Olaru, and P. Rodriguez-Ayerbe, "Convex lifting: Theory and control applications," Automatic Control, IEEE Transactions on (to appear). [Online]. Available: https://halcentralesupelec.archives-ouvertes.fr/hal-01326804/document

[28] N. A. Nguyen, S. Olaru, and P. Rodriguez-Ayerbe, "Any discontinuous PWA function is optimal solution to a parametric linear programming problem," in 54th IEEE Conference on Decision and Control, Osaka, Japan, 2015.

[29] _ - "Inverse parametric linear/quadratic programming problem for continuous PWA functions defined on polyhedral partitions of polyhedra," in 54th IEEE Conference on Decision and Control, Osaka, Japan, 2015

[30] N. A. Nguyen and S. Olaru, "A family of piecewise affine control Lyapunov functions," Automatica, vol. 90, pp. 212 - 219, 2018

[31] N. A. Nguyen, S. Olaru, P. Rodriguez-Ayerbe, M. Hovd, and I. Necoara, "Constructive solution of inverse parametric linear/quadratic programming problems," Journal of Optimization Theory and Applications, vol. 172, no. 2, pp. 623-648, 2017.

[32] N. A. Nguyen, S. Olaru, P. Rodríguez-Ayerbe, and M. Kvasnica, "Convex liftings-based robust control design," Automatica, vol. 77, pp. 206 - 213, 2017.

[33] R. Oberdieck and E. N. Pistikopoulos, "Explicit hybrid modelpredictive control: The exact solution," Automatica, vol. 58, pp. 152 - 159, 2015.

[34] E. N. Pistikopoulos, A. Galindo, V. Dua, E. S. Kikkinides, L. Papageorgiou, W. Jorisch, K.-W. Benz, W. Neumann, M. Kohler, W. Fritzsche, et al., Multi-Parametric Programming: Theory, Algorithms and Applications, Volume. Weinheim: WileyVCH, 2007.

[35] S. V. Rakovic and M. Baric, "Parameterized robust control invariant sets for linear systems: Theoretical advances and computational remarks," Automatic Control, IEEE Transactions on, vol. 55, no. 7, pp. 1599-1614, 2010.

[36] S. V. Rakovic, E. C. Kerrigan, K. I. Kouramas, and D. Q. Mayne, "Invariant approximations of the minimal robust positively invariant set," Automatic Control, IEEE Transactions on, vol. 50, no. 3, pp. 406-410, 2005.

[37] G. Valmorbida, L. Zaccarian, S. Tarbouriech, I. Queinnec, and A. Papachristodoulou, "Nonlinear static state feedback for saturated linear plants via a polynomial approach," IEEE Transactions on Automatic Control, vol. 62, no. 1, pp. 469-474, Jan 2017.

[38] M. Vatani, M. Hovd, and S. Olaru, "Control design for discrete-time bilinear systems using the scalarized schur complement," International Journal of Robust and Nonlinear Control, 2017.

[39] V. I. Zubov and L. F. Boron, Methods of AM Lyapunov and their application. Noordhoff Groningen, 1964. 